\title{
Hardware Monitors for Dynamic Page Migration
}

\author{
Mustafa M. Tikir ${ }^{\ddagger \S}$ \\ San Diego Supercomputer Center \\ 9500 Gilman Drive, 0505 \\ La Jolla, CA 92093 \\ mtikir@sdsc.edu
}

\author{
Jeffrey K. Hollingsworth \\ Computer Science Department \\ University of Maryland \\ College Park, MD 20742 \\ hollingsecs. umd.edu
}

$\ddagger$ This work was done while a student at the University of Maryland, College Park.
$\S$ Correspondence for the manuscript. Tel: (858) 822-0886 Fax: (858) 534-5117 


\begin{abstract}
In this paper, we first introduce a profile-driven online page migration scheme and investigate its impact on the performance of multithreaded applications. We use centralized lightweight, inexpensive plug in hardware monitors to profile the memory access behavior of an application, and then migrate pages to memory local to the most frequently accessing processor. We also investigate the use of several other potential sources of data gathered from hardware monitors and compare their effectiveness to using data from centralized hardware monitors. In particular, we investigate the effectiveness of using cache miss profiles, Translation Lookaside Buffer (TLB) miss profiles and the content of the on-chip TLBs using the valid bit information. Moreover, we also introduce a modest hardware feature, called Address Translation Counters (ATC) and compare its effectiveness with other sources of hardware profiles.

Using the Dyninst runtime instrumentation combined with hardware monitors, we were able to add page migration capabilities to a Sun Fire 6800 server without having to modify the operating system kernel, or to re-compile application programs. Our dynamic page migration scheme reduced the total number of non-local memory accesses of applications by up to $90 \%$ and improved the execution times up to $16 \%$. We also conducted a simulation based study and demonstrated that cache miss profiles gathered from on-chip CPU monitors, which are typically available in current microprocessors, can be effectively used to guide dynamic page migrations in applications.
\end{abstract}

\title{
Keywords
}

Dynamic Page Migration, Hardware Performance Monitors, cc-NUMA Systems, Multiprocessor Systems, OpenMP Applications, High Performance Computing, Address Translation Counters, Runtime Optimization, Full System Simulation 


\section{Introduction}

The dominant architecture for current shared-memory mult iprocessor systems is cache-coherent non-uniform memory access (cc-NUMA). In cc-NUMA systems, processors have a faster access to the memory units local to them compared to the remote memory units. For example, the remote and local latencies in midrange Sun Fire 6800 servers is around 300ns and 225ns[6], respectively where as the remote and local latencies in a 512 processor Altix 3000 is around 605ns and 145ns, respectively[21]. Traditionally cc-NUMA systems use physical first-touch page placement where memory pages are placed into the memory that is local to the processors that touch the page first. However, first-touch placement may result in non-local placement of a page relative to the processor that accesses it the most, which may have a significant impact on memory performance of the memory intensive applications running on cc-NUMA servers.

In this paper, we first introduce a user-level memory page migration scheme, namely dynamic page migration. In this page migration scheme, applications are profiled to determine the preferred locations of the memory pages in the memory units using hardware monitors. Then system calls are used to request the kernel to migrate the memory pages to the specific memory units. In this dynamic page migration scheme, both profiling and page migrations are conducted during the same run of the applications. The access frequencies of the memory pages by the processors are gathered continuously at runtime using hardware monitors and the pages are migrated local to the most frequently accessed processors at fixed time intervals.

Although page migration has been extensively studied in prior research, our dynamic page migration approach demonstrates several novel features. First, our goal is not to introduce a new page placement policy. Instead, we demonstrate that the combinations of in-expensive plug in hardware monitors that sample information about interconnect transactions and a simple page migration policy can be used effectively to improve the performance of real scientific applications. Second, even on multiprocessor systems with small remote to local memory latency ratios, optimizing page placement still provides substantial benefit to some applications. The remote to local memory latency ratio of the Sun Fire system we used is $1.33: 1$. We believe our page migration scheme will be more effective on systems with large remote to local latency ratios. 
The hardware performance monitors we used to gather page access profiles for our actual dynamic page migration scheme are centralized external pug-in monitors. These monitors listen to all address transactions on the system interconnect in the cc-NUMA server. However, such monitors are not available in most systems. Moreover, for non-bus based multiprocessors that do not use a common address and data bus, it is difficult to implement such centralized monitors that need to listen to all transactions on the system interconnect. Alternatively, many processors now include on chip hardware support for performance monitoring such as MIPS R10000[22], Compaq Alpha[7], Itanium from Intel[10], Sun UltraSPARC[17].

In this paper, we also investigate the use of several other potential sources of profiles gathered from hardware monitors in dynamic page migration and compare their effectiveness to using profiles from centralized hardware monitors. In particular, we investigate the effectiveness of using cache miss profiles and TLB miss profiles from on-chip CPU monitors, and sampling the content of the processor TLBs. If such sources of information can provide sufficiently accurate information, it would mean software based migration could be performed on systems without the need for dedicated hardware monitors. We also introduce a simple hypothetical modest hardware feature, called Address Translation Counters (ATC), which is specifically designed to gather profiles for dynamic page migration and compare its effectiveness with other sources of profiles. The ATC hardware is a set of additional counters included in the TLBs of a processor and gathers accurate information on access frequencies to the memory pages by the processor.

To evaluate the effectiveness of our dynamic page migration scheme, we implemented our page migration scheme for a Sun Fire 6800 server with Sun Fire Link[13] hardware monitors for the Sun Fireplane system interconnect. To evaluate the effectiveness of using each source of profiles in dynamic page migration, we conducted a simulation based study using a full system simulator, Simics[12]. We present the results of our studies in terms of the number of page migrations triggered, reduction in the number of nonlocal memory accesses, and improvement in execution times of the applications. We present the results for OpenMP C implementation of the NAS Parallel Benchmark suite[15] for both our actual page migration scheme and our simulation study. 


\section{Hardware and Software Components for Dynamic Page Migration Scheme}

In this section, we describe the hardware and software components used in our actual dynamic page migration scheme. We first describe the architecture of the Sun Fire servers. We next describe the centralized Sun Fire Link hardware monitors. Finally, we give a brief explanation about the system calls that we used.

\subsection{Sun Fire Servers}

The Sun Fireplane interconnect is Sun's fourth generation of Symmetric Multiprocessor Systems (SMP) interconnect. The Sun Fireplane interconnect is implemented with up to four levels of interconnect logic depending on the number of processors in the server[6]. In medium and large-sized Sun Fire servers, processors and memory units are grouped together on system boards (locality groups)[17]. Each system board contains 4 processors and 4 memory units local to the processors.

In Sun Fire servers, the transfer time to move a data block from a memory unit to the requesting device is non-uniform depending on the system boards the memory unit and requesting processor are on. Processors on a system board have faster access to the memory banks on the same board (local memory) compared to the memory banks on another board (non-local memory). For example, back-to-back latency measured by a pointer-chasing benchmark in a Sun Fire 6800 server with $750 \mathrm{MHz}$ CPUs is around $225 \mathrm{~ns}$ if the memory is local and $300 \mathrm{~ns}$ if the memory is non-local.

The Sun Fire 6800 server is a mid-range cc-NUMA architecture based on UltraSPARC III processors and Sun Fireplane interconnect. It supports up to 24 processors and 24 memory units. The processors and memory units in these servers are grouped into 6 system boards. Each processor has its own on-chip and external caches. Mid-range Sun Fire systems use a single snooping coherence domain that spans all the devices connected to a single Fireplane address bus.

\subsection{Sun Fire Link Hardware Monitors}

For our actual dynamic page migration scheme, we use the Sun Fire Link hardware monitors[14] to gather profiling information for page migration. The Sun Fire Link hardware monitor counts and samples the transactions on the address bus of the Sun Fireplane interconnect. These monitors were developed as part of a system to cluster multiple systems together, thus they listen to the address bus of the system interconnect. 
The Sun Fire Link Monitors consist of two 32-bit counter registers, a programmable control register that activates the counters, two registers to filter transactions based on transaction type, and two sets of mask and match registers to filter transactions based on other parameters, such as physical address range and the device identifier. In addition to counter registers, the Sun Fire Link Bus Analyzer has an 8-deep FIFO that records a limited sequence of consecutive interconnect address transactions. Each recorded transaction includes the requested physical address, the requestor device id, and the transaction type. The bus analyzer is configured with mask and match registers to select specific address ranges, processors or transaction types.

Even though the Sun Fire Link monitors provide useful information about the addresses and requesting processors, the information is at the level of physical addresses. To accurately evaluate the memory performance of an application, the address transactions have to be associated with virtual addresses used by the application. This requires us to reverse map physical addresses back to virtual addresses. We used the meminfo system call in Solaris 9 to create a mapping between physical and virtual pages in the applications.

\subsection{System Calls in the Solaris 9 Operating System}

To ensure the reusability of local caches in the processors, each application thread should be scheduled on the same processor, if possible, throughout its execution[16]. To ensure the reusability of local caches and to accurately count page access frequencies by processors independent of thread scheduling, we explicitly bind application threads to the processors in the system. We bind application threads to the processors in a round robin fashion using the processor_bind system call in Solaris.

Solaris places each physical memory page into the memory that is local to the first processor that touches the page. To move pages in our dynamic page migration scheme, we use the move-on-next-touch feature of the madvise system call in Solaris 9. Using the move-on-next-touch feature, we request the operating system to move a range of virtual memory onto the local memory of the processor that next touches the range.

\section{Dynamic Page Migration Methodology}

Our dynamic page migration algorithm consists of two different modules. The first module gathers profiling information using the Sun Fire Link monitors. The second module moves memory pages using the profiling information gathered by the first module. In our approach, we insert instrumentation code into the application 
to gather profiling information, to migrate the memory pages, to bind application threads to processors and to detect the application termination.

We used Dyninst[2] to insert instrumentation code into applications. Dyninst is a library that permits the insertion of code into a running program. The Dyninst library provides a machine independent interface to permit the creation of tools and applications that use runtime code patching.

For our dynamic page migration algorithm, instrumentation code is inserted at the entry of the main function, exit point(s) of thr_create function, and the entry of exit function. The instrumentation code that is inserted at main loads a shared library that creates additional helper threads for gathering profiling information and migrating memory pages. The instrumentation code inserted at the exit point(s) of thr_create calls the processor_bind system call to explicitly bind the newly created application threads to available processors in a round robin fashion. The helper threads are bound to dedicated processors and the remaining processors are used to bind the other threads in the application. The instrumentation code inserted at the entry to exit function detects the application termination and cleans up the hardware monitors.

Our dynamic page migration algorithm is a two-phase algorithm. It creates two helper threads, one for profiling and another for page migration. The profiling thread samples the interconnect transactions and updates the access frequencies of the memory pages for each system board. The migration thread stops the execution of all other application threads at fixed time intervals and triggers page migration based on the profiling information gathered. To trigger migration on a page, our scheme uses the move-on-next-touch feature of the madvise system call on the page. In addition, to prevent memory pages ping-ponging between memory units, we freeze memory pages that have been migrated recently for a fixed number of page migration iterations (We freeze a page for 3 consecutive iterations after migration). Thus, the memory pages are migrated at fixed time intervals and a page may be migrated more than once throughout application execution.

Our migration algorithm does not use a minimum access frequency threshold to trigger the migration of a page. At every migration interval, regardless of the number of accesses to a page, the page is considered as candidate for migration. Alternatively, we could limit migration to the pages with a minimum number of 
accesses or cache misses and thus migration overhead would potentially be eliminated for pages with little contribution to the application's memory time.

Our dynamic migration scheme does not have a particular mechanism for cache coherency but rather relies on the cache coherency mechanism the underlying operation system uses since our approach is designed for cache coherent NUMA systems that already have cache coherency mechanism implemented. Instead, we advise the underlying OS to move the page to a different location in physical memory and cache coherency is maintained by the OS by updating the TLB entries and invalidating the cache lines that are indexed using the physical addresses. Moreover, in our scheme, when migration is triggered for a page, we do not have a control whether a victim page will be evicted from the target physical memory if there is no available page for the migration to succeed. We instead rely on the mechanisms used in the underlying $\operatorname{OS}^{3}$.

In our page migration scheme, the two helper threads are bound to dedicated processors. However, these helper threads are mostly idle other than gathering profiling information and triggering page migrations at fixed time intervals. To isolate the impact of page migration on non-local memory accesses, we chose to bind these threads to dedicated processors. Instead, these threads could run on the processors the application threads run and make use of idle cycles. Alternatively, these threads can be pushed to the OS level by adding two more threads to the OS. More importantly, considering the impact of chip-level multiprocessor architecture on processor costs, additional dedicated processors can be included to the HPC systems for application profiling and page migration.

\section{Other Sources of Hardware Profiles for Dynamic Page Migration}

In our actual page migration scheme on the Sun Fire server, we use the centralized Sun Fire Link monitors to identify the preferred locations of memory pages for dynamic page migration. However, such monitors are not available in many systems. Moreover, for non-bus based multiprocessors that do not use a common address and data bus, it is difficult to implement such centralized monitors that need to listen to all

${ }^{3}$ In the underlying OS we used for this research, page migration fails if there is no available physical page on the target memory. However, we have not seen a case where migration was denied since we track how much memory is used by the application and don't move pages if we run short on memory. In practice, this constraint never caused us to fail to make a desired migration. 
transactions on the system interconnect. Alternatively, many processors now include on chip hardware monitors for performance tuning. In this section, we describe other potential sources of profiles that can be used to generate page access frequencies. Later in the experiments section, we present the results of our simulation based study to investigate the effectiveness of these other sources of profiles.

\subsection{Profiles from Distributed On-Chip CPU Monitors}

Profiles of page access frequencies by processors in an application running on a cc-NUMA system can be gathered by using information about the cache or TLB misses by each processor in the system. If the information about the number of cache or TLB misses on each page by a processor is known, the access frequency of the page by the processor can be approximated. However, for such information to be available, the addresses associated with the cache and TLB misses are needed.

Many processors include hardware support to count events for performance monitoring. Moreover, they often provide mechanisms to trigger an interrupt when a given number of events occur. More recently, an increasing number of processors provide the ability to capture the memory addresses and/or instructions involved in performance critical events. (Note that some monitors may provide approximate information about the instruction(s) involved due to the difficulty of associating information with specific events when many instructions are in flight. However, even approximate information still provides valuable insight for dynamic tuning of applications). For example, the Itanium 2 processor provides a set of event address registers (EARs) that record the instruction and data addresses of data cache misses, the instruction and data addresses of data TLB misses, and the instruction addresses of instruction TLB and cache misses[10]. Thus, by distributed sampling of the addresses associated with the cache or TLB miss events, profiles of page access frequencies by processors can be generated. Moreover, since cache miss events are generally distributed throughout the execution and provide information on fine grain behavior, profiles of page access frequencies gathered from cache miss events may be more representative. Compared to cache misses, the number of TLB miss events is generally lower and these events may not correspond to the pages that are frequently accessed due to the fact that applications tend to keep frequently accessed pages in TLBs. In this paper, we investigate the use of cache and TLB miss information from on-chip CPU hardware. 


\subsection{Profiles from Valid Bit Information in TLB Entries}

Hardware tries to keep virtual-physical page translation entries of the frequently accessed pages in the processor TLBs. Thus, the contents of the valid TLB entries in a processor potentially provide information on the pages that are mostly accessed by the processor. By sampling the content of the TLBs periodically, it is possible to approximate page access frequencies by the processor. Similarly, the information from each processor can be combined and page access frequencies by processors can be generated to guide migrations in a dynamic page migration scheme.

To sample the contents of valid TLB entries of a processor, the underlying operating system needs to provide a software sampling mechanism. In particular, the operating system needs to provide a means to query the list of valid entries and the virtual addresses of the pages for each valid TLB entry. In our research, we assume the underlying operating system provides a system call that returns the list of virtual page addresses in the valid TLB entries for a given processor.

\subsection{Address Translation Counters}

To further evaluate the effectiveness of sources of profiles in dynamic page migration, we designed a dedicated hardware monitor that gathers accurate page frequencies and compared the effectiveness of other sources of profiles with the dedicated monitors.

The hypothetical hardware feature we use, Address Translation Counters (ATC), is a set of additional counters that is included in the TLBs of the processors. In ATC, a counter is included for each TLB entry in a processor (Shown in Figure 1) and incremented when a virtual to physical address translation is satisfied by the corresponding TLB entry. Moreover, when the content of a TLB entry is evicted due to a TLB miss or invalidated due to other reasons such as cache coherency operations, the counter associated with the TLB entry is cleared. The ATC is included in each processor and counts the number of accesses to the memory pages by the processor using the virtual to physical address translations requested while the memory pages are actively accessed. Note that the main reason for introducing these counters is to evaluate the effectiveness of other sources of profiles by comparing them to this hardware feature as ATC counters are more likely to capture page access frequencies more accurately compared to other more indirect sources of profiles. 
Associating information hardware monitors provide with specific events with many instructions in flight can be difficult, but that associating TLB misses and ATC events with memory addresses is relatively easy due to the fact that virtual page addresses are already stored in the TLB and that TLB misses must be necessity to update the TLB.

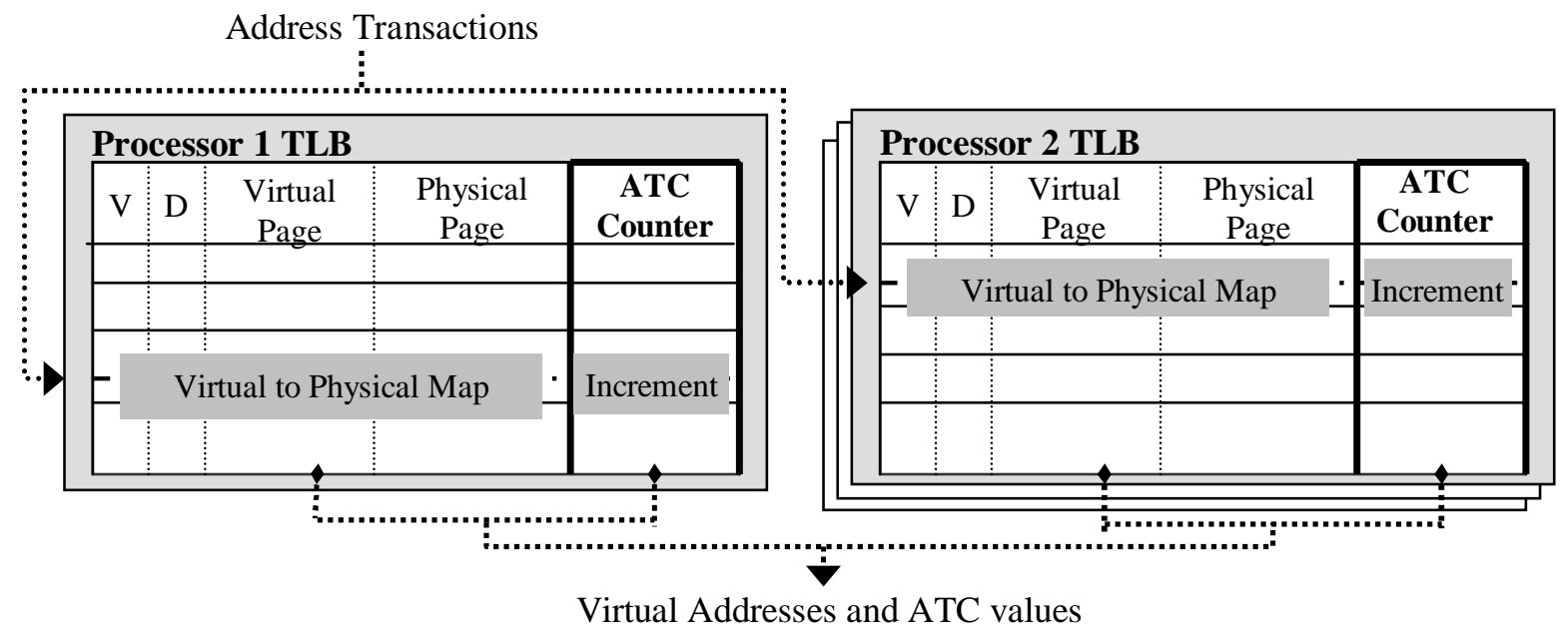

Figure 1. Information flow in the Address Translation Counters

Information recorded by the ATC hardware can be gathered in several ways. One way is to sample the content of the counters regularly during execution along with the virtual page addresses associated with these counters. Another approach is that the operating system may provide low-overhead software traps such that when a TLB entry in invalidated due to a TLB miss or cache-coherency operation, the content of the corresponding ATC counter value and the associated virtual page address can be provided to the application (similar to the software TLB miss handler in MIPS processors[9]). Lastly, the underlying operating system could include an additional field for each page table entry where the ATC entry can be saved at context switches. Later, the count information can be gathered via system call by querying the page table content. For our research, we assume the underlying operating system provides means to sample ATC content.

\section{Experimental Results}

In this section we first present the results of experiments we conducted to ensure that we could accurately sample address transactions via hardware monitors in the applications being analyzed. We then present the results of experiments in which we evaluated our actual dynamic page migration scheme on the real 
hardware and simulation based study where we compared the effectiveness of other sources of profiles to the centralized plug in hardware monitors we used in our actual migration scheme.

\subsection{Interconnect Transaction Sampling Experiments}

We sample the interconnect transactions using hardware monitors and approximate the access frequencies for the memory pages. However, for sampling to be effective, the sampling technique has to be representative of all transactions that occurred during the execution of the application being analyzed.

One approach to sample interconnect transactions via hardware monitors is to continuously sample at the maximum speed of the interconnect instrumentation software. We refer to this sampling scheme as maximum-rate sampling. Maximum-rate sampling does not capture a compete set of transactions, but it tries to sample as many transactions as possible. Alternatively, transactions can be sampled at fixed time intervals or at every $N^{\text {th }}$ transaction occurrence, where $\mathrm{N}$ is a constant that defines the interval of sampling[3]. We refer to sampling at every $N^{\text {th }}$ transaction occurrence as interval sampling.

We conducted a series of experiments to compare how representative the maximum-rate and interval sampling techniques are of all transactions. To objectively compare the two sampling techniques we designed a distance metric $D$ that given a set of transactions and a set of samples from the set, measures the percent difference between the values of a property for these sets. The property we used in our experiments is the ratio of transactions requested by a specific processor to the total number of transactions. This metric indicates how much a set of transactions deviate from another set of transactions in terms of memory behavior. Thus, the closer the value of our distance metric is to 0 , the more representative the set of sampled transactions is of the set of all transactions.

For this study, we used the Sun Fire Link counters. Since the Sun Fire Link counters can accurately count the number of transactions as well as the number of transactions from a given processor, we counted both of these values and compared them with samples taken via Sun Fire Link bus analyzer to approximate the sampling error of sampling techniques.

For each experiment, we configured one of the two counters in the Sun Fire Link hardware monitors to count the number of transactions requested by a selected processor $P$, denoted $C_{P}$. The other counter is 
configured to count all transactions, $C_{A}$. Using the Sun Fire Link bus analyzer we also sampled interconnect transactions and recorded the number of transactions sampled, denoted $S_{A}$. In the set of sampled transactions, we count the number of transactions that are requested by processor $P$, denoted $S_{P}$. We calculate the ratios for the set of sampled transactions and the set of all transactions as $R_{\text {Sample }}=S_{P} / S_{A}$ and $R_{A l l}=C_{P} / C_{A}$, respectively. We define the distance as $D=A B S\left(R_{\text {Sample }}-R_{\text {All }}\right) / R_{A l l}$. That is, the distance metric gives an insight as to how far the set of sampled transactions deviate from the set of all transactions.

We conducted a series of experiments for a set of processors while running an OpenMP version of the CG benchmark from NAS Parallel benchmark suite[15]. We ran CG with 6 threads using the input set of size B. We repeated the experiments with different sampling intervals in which samples taken at every 64,256 , 1024 and 4096 transactions.

Table 1 presents the results of the experiments conducted to compare how representative the sampled transactions are of all transactions with respect to our distance measure. In Table 1, the second column gives the distance values for maximum-rate sampling, the third to sixth columns give results for interval sampling with different interval values. The rows that are labeled with processor identifiers give the distance between the set of all transactions and the set of sampled transactions with respect to that processor.

\begin{tabular}{|l|c|c|c|c|c|}
\hline \hline & Max-Rate & \multicolumn{4}{|c|}{ Interval Sampling } \\
\cline { 3 - 6 } & Sampling & $\mathbf{4 K}$ & $\mathbf{1 K}$ & $\mathbf{2 5 6}$ & $\mathbf{6 4}$ \\
\hline \hline Processor 0 & 0.51 & 0.03 & 0.03 & 0.03 & 0.09 \\
Processor 1 & 0.61 & 0.04 & 0.04 & 0.04 & 0.09 \\
Processor 2 & 0.47 & 0.01 & 0.02 & 0.02 & 0.23 \\
Processor 3 & 0.58 & 0.00 & 0.01 & 0.01 & 0.02 \\
Processor 4 & 0.65 & 0.02 & 0.02 & 0.02 & 0.12 \\
Processor 5 & 0.57 & 0.03 & 0.02 & 0.03 & 0.15 \\
\hline \hline Average Dist. & 0.56 & 0.02 & 0.02 & 0.02 & 0.11 \\
\hline \hline \% Sampled & 17.56 & 0.19 & 0.78 & 3.07 & 9.75 \\
\hline \hline
\end{tabular}

Table 1. Distance values for maximum-rate sampling and interval sampling

Table 1 shows that even though the maximum-rate sampling can sample about $18 \%$ of all transactions, the distance metric is significantly higher compared to interval sampling for all processors. Moreover, for maximum-rate sampling, the average distance over all processors is 0.56 , which shows that the set of sampled transactions is quite different from the set of all transactions. 
During maximum-rate sampling, the maximum number of transactions the instrumentation software can record bounds the number of samples that can be taken for a processor. Thus, if a processor requests transactions faster than the maximum rate the instrumentation software can read, many transactions for the processor are not recorded. Similarly, if a processor requests transactions slower than the rate of instrumentation software, almost all of its transactions will be recorded as samples. Thus, maximum-rate sampling results in a skewed distribution of sampled transactions with respect to the level of memory system activity on processors and the sample set does not accurately represent all transactions.

Table 1 also shows that for interval sampling, the distance values depend on the sampling rate. The distance values are low and similar to each other except for the experiments where transactions are sampled at every 64 transactions. In particular, if the samples are taken at every 256 transactions or more, the set of sampled transactions is fairly representative of all transactions. Table 1 also suggests that if the rate of interval sampling exceeds $5 \%$ of all transactions, the set of sampled transactions becomes less representative.

To further investigate how representative the samples for larger sampling interval values, we also conducted experiments varying the sampling interval up to every-128M address transactions. In addition, for each experiment, we also recorded the number of distinct pages that are included in the set of sampled transactions. Figure 2 presents the average sampling error (left y-axis) and the percentage of distinct pages sampled (right y-axis) in the application for the intervals we tested.

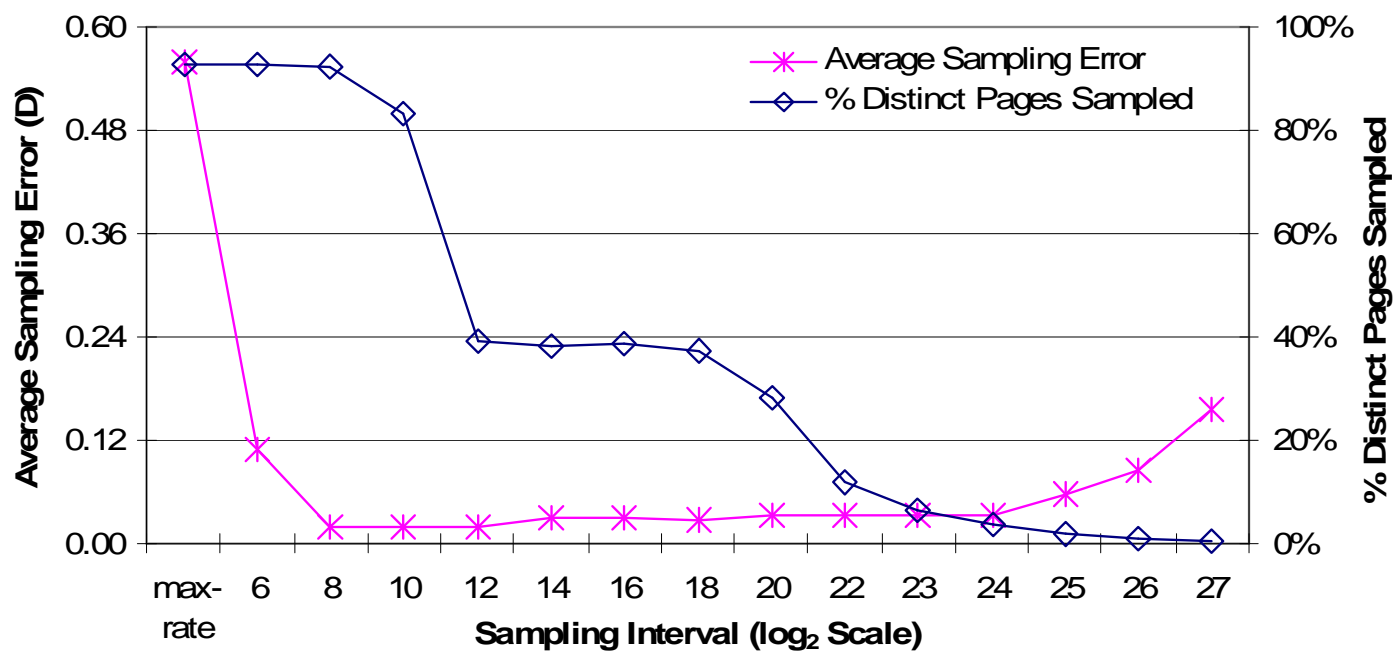

Figure 2. Average distance and percentage of pages sampled in CG (B) 
Figure 2 shows that average sampling error is the highest for max-rate sampling and it starts decreasing dramatically as the sampling interval increases. Moreover, average error stays low and steady for a large range of sampling intervals starting at every-256 transactions sampling to every-8M transactions sampling. Average sampling error starts to increase again after every-16M transaction sampling due to the fact that the number of samples taken is not large enough to accurately characterize all transactions in the application.

Figure 2 also shows that for max-rate sampling, 93\% of all pages in the application are included in the samples taken. Similarly, for smaller intervals, the percentage of distinct pages sampled is around $90 \%$ for interval sampling. However, as the sampling interval increases, Figure 2 shows that the percentage of distinct pages sampled in interval sampling decreases dramatically, resulting in many pages not included in the set of sampled transactions. Figure 2 shows that even though interval sampling generates more representative samples, the percentage of the pages included in the samples decreases as the sampling interval increases.

\subsection{Page Migration Experiments}

To investigate the effectiveness of our actual dynamic page migration scheme on the performance of real applications, we conducted experiments using the OpenMP C implementation of the NAS Parallel Benchmark suite[15]. We chose applications with different sizes ranging from B to C (large data set sizes). We compiled the applications using Sun's native compiler, Sun C 5.5 EA2, with optimizations (xopenmp=parallel and -O3) on to support parallelized code.

We conducted all of our experiments on a 24-processor Sun Fire 6800 with $24 \mathrm{~GB}$ of main memory. The memory in each system board is 8-way interleaved where each processor controls two banks of memory. The Sun Fire Link hardware is plugged into an I/O drawer in this system. The Sun Fire Link instrumentation has full visibility into all transactions on the Fireplane interconnect.

To quantify the benefits of our dynamic page migration approach, we conducted a series of experiments with and without page migration. For all applications, we measured both the original execution times and the execution times when pages are migrated using our dynamic page migration approach. For each application, we also measured the percentage reduction in the number of non-local memory accesses when memory pages 
are dynamically migrated compared to its original execution. We sampled interconnect transactions at every 1024 transactions for experiments with page migration.

We ran all applications with 12 threads on 6 system boards of the Sun Fire 6800 server where each board executed 2 threads rather than running the applications with 24 threads where each processor is assigned a thread. This is due to the fact that we noticed a form of intra-board locality in the Sun Fire servers [18] that can mislead the benefits of page migration in isolation. We observed the choice of the processor from the group of processors on the same system board can also have an impact on the execution times of applications. We implemented a simple benchmark and measured the execution time of this benchmark when different processors in the same system board are used to execute the application. In each execution, to eliminate the effect of memory page placements, all memory pages in the benchmark are explicitly placed locally. We observed that our simple benchmark took up to $11 \%$ more time to execute when it is bound to the second processor of the system board compared to when it is bound to the first processor even though the array pages are placed local to the processors [18]. The intra-board variations can be partially explained by resource sharing contention among processors, bookkeeping operations running on processors by OS and whether the array pages are placed on the memory banks controlled by the processor running the application or on the memory banks controlled by another processor in the same system board. To eliminate any possible contention due to resource sharing among processors, we scheduled only two threads on each system board rather than four threads such that we would isolate the benefits of page migration alone for our experiments and the gain due to the page migrations is not overcome by the intra-board variations.

As explained in Section 3, we insert instrumentation code into the application using the Dyninst library. For each application, the instrumentation overhead is a one-time overhead since the Dyninst library has a capability of saving instrumented executables for later reuse. Moreover, the instrumentation overhead for our page migration approach is independent from the execution times of the applications we analyzed. We measured the instrumentation overhead for all applications for our dynamic page migration approach and it is typically around 2 seconds. 
For the experiments with page migration, the migration interval is given as a parameter to our dynamic page migration scheme. To investigate the impact of migration intervals and choose the migration interval for the experiments, we conducted a sensitivity analysis in which we ran each application under different migration intervals ranging from 1 second to 50 seconds. Our experiments showed that migration interval used does not have a major impact on the performance of the applications except MG. For MG, migration interval has a significant impact due to the fact that MG is a short running program and when migration is triggered at a slower rate, MG does not benefit from page migrations. Thus, for our page migration experiments, we chose to trigger page migration at every 5 seconds. We chose 5 seconds as the migration interval such that we would trigger enough number of migrations in all applications to benefit from dynamic page migration but still keep a slower rate of migrations in the other applications for a lower overhead.

\subsubsection{Reduction in Non-Local Memory Accesses due to Page Migrations}

To quantify the benefits of our dynamic page migration approach, we counted the total number of non-local memory accesses for all applications with and without using dynamic page migration. We used the Sun Fire Link hardware monitors to measure the total number of non-local memory accesses in the applications.

Table 2 presents the percentage reduction in the total number of non-local memory accesses when dynamic page migration is used compared to when memory pages are not migrated. In the second column, we give the total number of address transactions requested by each application during its execution. The third column gives the percentage of non-local memory accesses without our page migration approach and the fourth column shows the percentage of non-local memory accesses when memory pages in the application are migrated using our dynamic page migration approach. The fifth column lists the percentage reduction in the total number of non-local memory accesses when dynamic page migration is used.

Table 2 shows that for all applications, our dynamic page migration approach was able to reduce the number of non-local memory accesses by $19.7-89.6 \%$ (The average reduction for applications is $58.3 \%$ ). Table 2 also shows that for MG, a significant number of non-local memory accesses were eliminated when memory pages were migrated. This is due to the fact that the first-touch policy in the underlying operating 
system placed pages poorly in a single memory unit and our migration policy was able to migrate pages to several memory units according to their access pattern.

\begin{tabular}{|l|r|r|r|r|}
\hline \hline & \multirow{2}{*}{$\begin{array}{c}\text { \# of Address } \\
\text { Transactions } \\
\text { (Millions) }\end{array}$} & \multicolumn{2}{|c|}{$\begin{array}{c}\text { Percentage of } \\
\text { Non-local }\end{array}$} & \multicolumn{2}{c|}{$\begin{array}{c}\text { Accesses } \\
\text { w/o Page } \\
\text { Migration }\end{array}$} & $\begin{array}{c}\text { Page } \\
\text { Migration }\end{array}$ & \\
\cline { 3 - 4 } Reduction \\
BT (B) & 38,507 & 40.9 & 25.3 & 38.0 \\
CG (C) & 15,721 & 80.9 & 15.3 & 81.0 \\
EP (C) & 42 & 85.4 & 28.2 & 67.0 \\
FT (B) & 2,329 & 64.2 & 29.6 & 54.0 \\
LU (C) & 48,682 & 41.2 & 33.1 & 19.7 \\
MG (B) & 841 & 80.5 & 8.3 & 89.6 \\
SP (C) & 116,116 & 55.0 & 22.7 & 58.8 \\
\hline \hline
\end{tabular}

Table 2: Reduction in non-local memory accesses due to page migration

Unlike MG, for LU our dynamic page migration approach was not able to reduce the number of nonlocal memory accesses significantly. For LU, first-touch policy placed memory pages better. Moreover, system boards uniformly access the majority of the memory pages that our dynamic approach was able to migrate. That is, while migrating those pages to a system board reduces the number of non-local memory accesses requested by the processors in that system board, the number of non-local memory accesses by the processors in all other system boards increases. Our dynamic page migration approach uses a simple decision mechanism that identifies the preferred location of a memory page as the system board that accesses it most. It does not take the access frequencies by other system boards into consideration. The access frequencies by other system boards may also be used to better decide whether a page should be migrated[19].

\subsubsection{Impact of Page Migration on Cache Usage}

The UltraSPARC III processors in the Sun Fire servers use physical addresses to index their external caches. Since page migration changes the physical addresses of the memory pages in an application, it is also necessary to ensure that our page migration approach does not have a significant impact on the cache behavior of applications. To quantify the cache usage of the applications, we counted the number of conflict and capacity misses (i.e. non-compulsory misses) during the execution of the applications with and without dynamic page migration using the Sun Fire Link monitors. The Sun Fire Link monitors measure noncompulsory misses by measuring the number of write-back (WB) transactions requested. A WB transaction 
is requested when a dirty cache line is evicted from the external cache due to a capacity or conflict miss. Table 3 presents the number of WB transactions with and without our page migration approach.

\begin{tabular}{|l|r|r|c|}
\hline \multirow{2}{*}{} & \multicolumn{2}{|c|}{ \# of WB Transactions (Millions) } & \multirow{2}{*}{ \% Change } \\
\cline { 2 - 3 } & $\begin{array}{c}\text { w/o Page } \\
\text { Migration }\end{array}$ & Page Migration & \\
\hline \hline BT (B) & $14,948.8$ & $14,900.1$ & -0.33 \\
CG (C) & 270.6 & 268.7 & -0.67 \\
EP (C) & 12.3 & 12.6 & 2.38 \\
FT (B) & 855.0 & 851.8 & -0.37 \\
LU (C) & $18,252.8$ & $18,171.6$ & -0.44 \\
MG (B) & 217.4 & 218.0 & 0.28 \\
SP (C) & $39,223.3$ & $39,139.9$ & -0.21 \\
\hline \hline
\end{tabular}

\section{Table 3: Percent change in the number of write-back transactions}

Table 3 shows that our dynamic page migration approach does not have a significant affect on the cache behavior of applications. It also shows that our dynamic page migration approach has a higher impact on EP compared to other applications. However, EP does not allocate a significant number of memory pages and thus the absolute number of cache misses is more than a factor of 20 lower than any other application we measured. Moreover, since the working set of EP fits in local caches, the increase in cache misses in EP is mainly due to the invalidation of cache lines caused by migration of memory pages.

\subsubsection{Execution Times with Page Migration}

While reducing the number of non-local memory accesses in an application is important, what matters is the impact of this reduction on application's runtime. Thus, we measured the impact of our page migration approach on the execution times of the applications. For each application, we conducted three different experiments and measured the total execution time for each experiment.

First, we ran each application using our dynamic page migration approach and measured the total execution time including overhead due to the creation of the helper threads and triggering page migrations. Even though the migration thread runs in parallel with other threads of the application, it suspends all application threads to trigger the actual page migrations and later resumes their executions. During the second set of experiments, we measured the original execution times of the applications with no intervention. Lastly, we conducted a third set of experiments to investigate the impact of binding application threads to 
fixed processors, and therefore the impact of dynamic page migration in isolation. During these experiments, we ran each application with page migration disabled but bound the threads to the processors in the system.

For each application and experiment, we repeated the experiment seven times and recorded the minimum of the execution times among all runs. We used the minimum execution time since we noticed higher variation in the original execution times for some applications. We suspect the higher variation in the original execution times of those applications is due to differences in the initial page placements and thread scheduling by the operating system.

Table 4 presents the execution times of the applications we analyzed. The second column lists the original execution times of the applications. In the third column, we present the execution times when the application threads are bound to the processors throughout the executions. The fourth column lists the execution times of the applications when pages are migrated using our dynamic page migration approach. The fifth column presents the number of page migrations triggered. Lastly, the sixth column presents the overhead due to page migrations.

Table 4 shows that for all applications except LU and MG, when the application threads are bound to processors the applications run faster by $0.16-1.76 \%$ compared to their original executions. However, LU slows down by $0.6 \%$ where MG slows down by $2.2 \%$ when their threads are bound to the processors. Table 4 shows that binding application threads to the processors is almost always beneficial even though the performance gain is not significant.

\begin{tabular}{|l|r|r|r|r|r|}
\hline & \multicolumn{2}{|c|}{ Execution Times (seconds) } & \multirow{2}{*}{$\begin{array}{c}\text { \# of } \\
\text { Migrations }\end{array}$} & $\begin{array}{c}\text { Overhead } \\
\text { (seconds) }\end{array}$ \\
\cline { 2 - 4 } & Original & $\begin{array}{c}\text { Bound } \\
\text { Threads }\end{array}$ & $\begin{array}{c}\text { Page } \\
\text { Migration }\end{array}$ & & 11.8 \\
\hline BT (B) & 996 & 992 & 966 & 112,310 & 4.4 \\
CG (C) & 625 & 613 & 534 & 47,213 & 0.3 \\
EP (C) & 293 & 292 & 292 & 2,071 & 15.1 \\
FT (B) & 113 & 112 & 118 & 177,602 & 13.1 \\
LU (C) & 1981 & 1994 & 1978 & 132,696 & 2.7 \\
MG (B) & 31 & 32 & 26 & 49,884 & 17.1 \\
SP (C) & 3901 & 3854 & 3347 & 138,943 & \\
\hline \hline
\end{tabular}

Table 4: Execution times, number of migrations and migration overhead 
Table 4 also shows that the overhead due to page migration is mainly proportional to the number of page migrations requested and it ranges up to $12.8 \%$ compared to the original execution times of the applications. To guarantee that the migration thread touches the page next before other threads, all other threads have to be suspended. If the operating system instead provided a system call that would allow applications to indicate the target locations of the memory pages, it would permit migration of pages to their target locations during the next available opportunity, and thus partially reduce the page migration overhead.

Figure 3 presents the performance improvement when our page migration approach is used compared to both the original execution time and the execution time when the threads of the applications are bound to processors. Under the label of each application on the x-axis, Figure 3 also presents the migration overhead percentage with respect to the original execution time of the application. The migration overhead includes time spent for the suspension of all threads and moving pages to their target memory location. Figure 3 shows that our dynamic page migration approach was able to improve the execution performance of the applications except FT by up to $15.9 \%$ compared to their original executions. However, FT runs slower under dynamic page migration.

Our dynamic page migration approach improved the performance of CG and SP by $14.5 \%$ and $14.2 \%$, respectively, compared to their original execution times. CG and SP request many memory accesses and our dynamic page migration approach was able to eliminate many of the non-local memory accesses (see Table 2). In addition, dynamic page migration improved the execution performance of CG and SP by $12.8 \%$ and $13.2 \%$ respectively, compared to the executions where application threads are bound.

Like CG and SP, our dynamic page migration approach was also able to improve the performance of MG by $15.9 \%$ compared to its original execution time. Even though MG does not request many memory accesses, our page migration approach was still able to reduce the number of non-local memory accesses significantly (see Table 2). Compared to the execution of MG when its threads are bound to the processors, dynamically migrating memory pages in MG improved the execution performance by $18.1 \%$.

Figure 3 also shows that our dynamic page migration approach improved the execution performance of BT by $2.9 \%$ compared to its original execution and by $2.6 \%$ compared to execution where its threads are 
bound to processors. Figure 3 also shows that our page migration approach is not as effective for BT as for $\mathrm{CG}, \mathrm{MG}$, and SP, which is partially due to fact that the reduction in the number of non-local memory accesses in BT is not as high. Similarly, our page migration approach improved the performance of LU by $0.8 \%$, which is also mainly due the small reduction in number of non-local memory accesses.

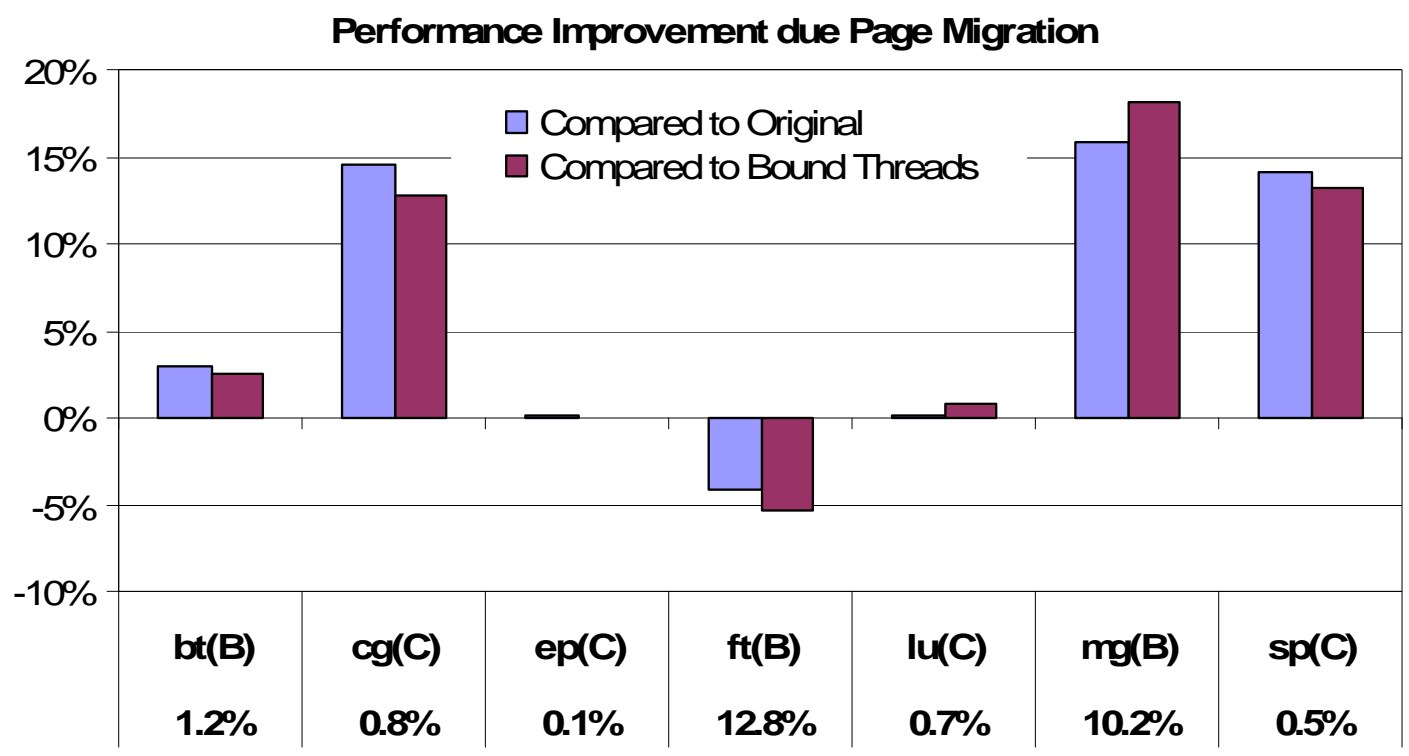

Figure 3: Performance gain for the applications under dynamic page migration. The percentages under each application label present the migration overhead percentage with respect to the original execution time of the application.

Figure 3 also shows that our dynamic page migration approach was not as effective in improving the execution performance of EP even though it reduced the number of non-local memory accesses by $67.0 \%$. EP reuses data in the local caches of the processors, and the majority of its memory accesses are requested at the beginning of its execution, before the memory pages are migrated.

Figure 3 shows that our dynamic page migration approach was not able to improve the execution performance of FT even though it reduced the number of non-local memory accesses in FT by $54.0 \%$. Instead, our page migration approach slowed down the execution of FT by around $4.2 \%$ compared to its original execution. However, Figure 3 also shows that the slowdown for FT is mainly due to the overhead introduced by page migration, which is $12.8 \%$ of the original execution time for FT. That is, the reduction in the number of non-local memory accesses did not overcome the overhead introduced by migration of many pages that are initially placed poorly. Moreover, the page migration overhead for FT would be partially 
reduced if the operating system did not require suspending application threads to trigger the actual migrations by touching pages and instead provided a mechanism to directly request migration.

Overall, our dynamic page migration approach reduced the total number of non-local memory accesses of applications by up to $90 \%$ and improved the execution times up to $16 \%$. To investigate the effectiveness of our approach, however, we also conducted experiments to measure how dynamic page migration compares to ideal static page placement in terms of reduction in non-local memory accesses and performance improvement. We modified our scheme to run in two phases where during the first phase, profiling information is collected and during the second phase, application is run with ideal static placement where pages are placed at application start using the profiling information and move-on-next touch feature of the underlying OS. Even though our experiments showed that ideal static page placement also resulted in a reduction of non-local memory accesses compared to the original execution, it was not as effective compared to our dynamic page migration scheme. For profile-driven static page placement, reduction in non-local memory accesses was $28.6 \%$ less in the average compared to using dynamic page migration except FT. For FT, ideal static page placement was able to reduce non-local memory accesses up to $68 \%$ where the reduction is only $54 \%$ when dynamic page migration is used (Note that this partially explains why dynamic page migration overhead is higher for FT.). More importantly, the improvement in performance of applications was only $0.02-7.9 \%$ when static page placement is used, indicating that the dynamic page migration is more effective in both reducing the number non-local memory accesses and improving the execution performance of applications compared to using profile-driven static page placement scheme.

To briefly investigate the impact of suspending all application threads on the overall migration overhead, we also ran experiments where application threads were kept running hoping that the right processor touches the pages that are to be migrated next. This would also give us some insight on the benefits of using an operating system with a system call that does not require suspension of application threads for page migration. However, our experiments showed that the reduction in the number of non-local memory was not as high when application threads are not suspended and resulted in 17-26\% more non-local memory accesses compared to when application threads are suspended. This is mainly due to the fact that majority of the pages 
are accessed by multiple processors throughout the execution (even though with different frequencies) and some of the pages for migration are touched by other process ors than the one running on its target location. More importantly, the reduction in the overall migration overhead was insignificant compared to original the execution times. Thus, Table 4 and these results indicate that the overhead of actual moving pages to their target locations (between two physical memories) dominates the overall migration overhead. Hence, a new system call that would not require suspension of threads to correctly migrate pages to their preferred locations would only reduce the overall migration overhead by a small amount.

\subsubsection{Graphical User Interface for Dynamic Page Migration}

To visualize the page placement in the applications, we implemented a Graphical User Interface (GUI) that presents the locations of the virtual memory pages in terms of the memory units (system boards) in the underlying CC-NUMA server. Our dynamic page migration GUI also presents additional information such as the number of page migrations triggered for each migration interval, the stack percentage bars indicating the percentages of pages migrated to each memory unit for the latest migration interval.

Figure 4 shows the GUI snapshot for application MG when dynamic page migration is not used. The bottom window in Figure 4 visualizes the virtual address space of the application where each pixel (or a sequence of pixels when a portion of application's address space is displayed) represents a virtual page and the color of the pixel presents the memory unit the page is placed. The virtual page index increases from left to right and top to bottom, starting with the page index 0 at top left corner of the window. Note that in our GUI, there are 6 colors to represent the locations of the pages due to the fact the Sun Fire 6800 server we used have 6 memory units (boards).

Figure 4 shows that almost all of the memory pages in MG are placed in a single memory unit when MG is run without page migration. This is due to the fact that MG starts with a single thread that initializes its data structures, hence first-touch placement in the underlying operating system places pages in the memory unit on the same board as the initialization thread runs.

Figure 5 shows the GUI snapshot for MG when run with dynamic page migration (every 5-second migration) after several migration iterations. Figure 5 shows that our dynamic page migration scheme was 
able to accurately move pages local to the processors accessing them most. Even though Figure 5 shows some imperfections in the placement of the pages due page migration, it clearly indicates the stride-access pattern in MG. We believe imperfections are caused by the fact that information on some memory pages is not included in the profiles gathered from performance monitors due to use of interval sampling.

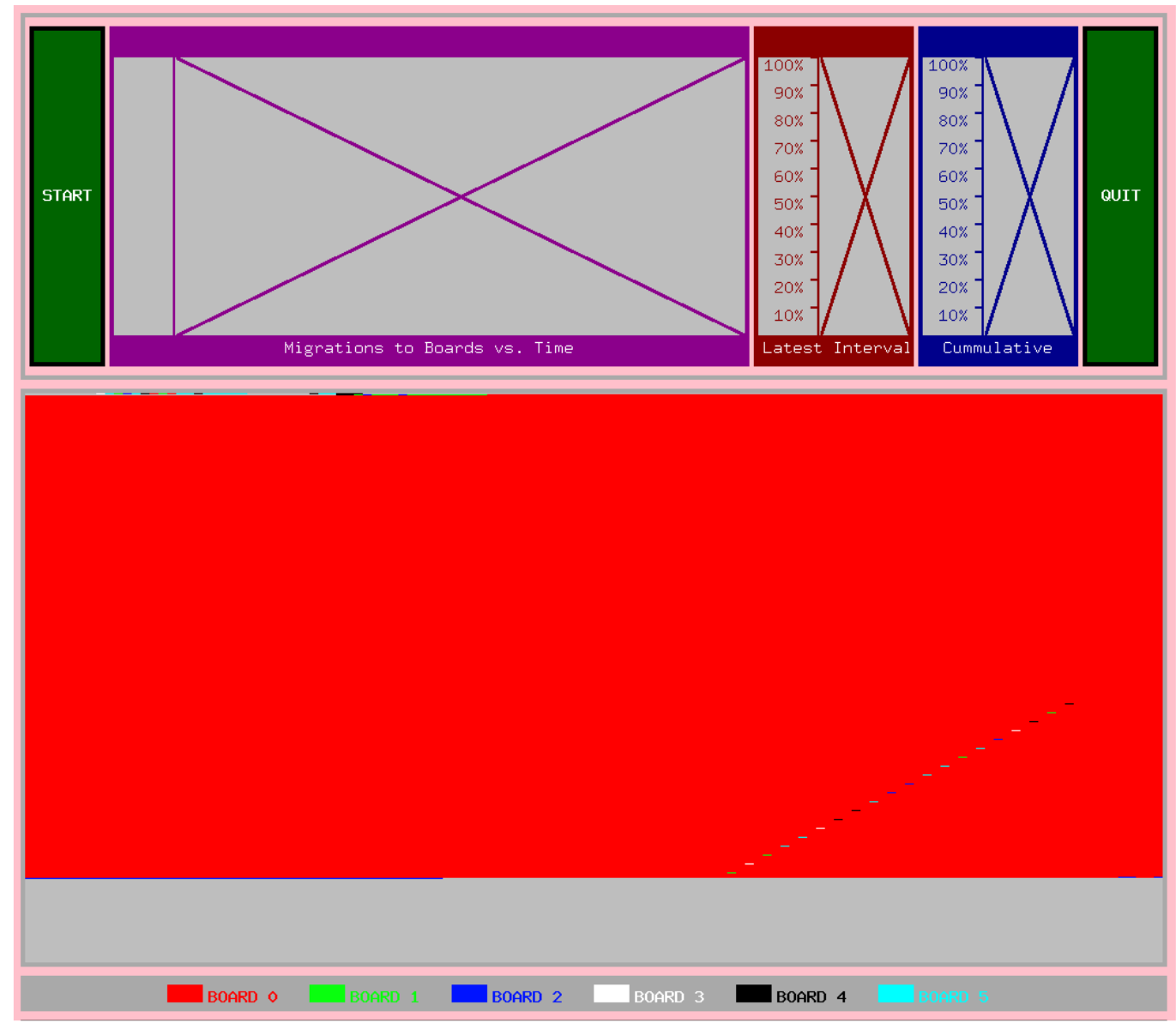

Figure 4: GUI snapshot for page placement in MG without page migration

In addition to locations of the memory pages in an application, our dynamic page migration GUI also presents detailed information on page migrations triggered when run with dynamic page migration. The window at the top-left corner in Figure 5 displays the number of page migrations triggered to each memory unit for each migration interval. The top middle window displays the stack percentage bar that presents the percentages of migrations triggered to each memory unit for the latest migration interval in addition to the total number of pages migrated for the interval. Similarly, the top right window displays the stack percentage bar that presents the percentages of migrations triggered to each memory unit since the application start in addition to the total number of pages migrated. 


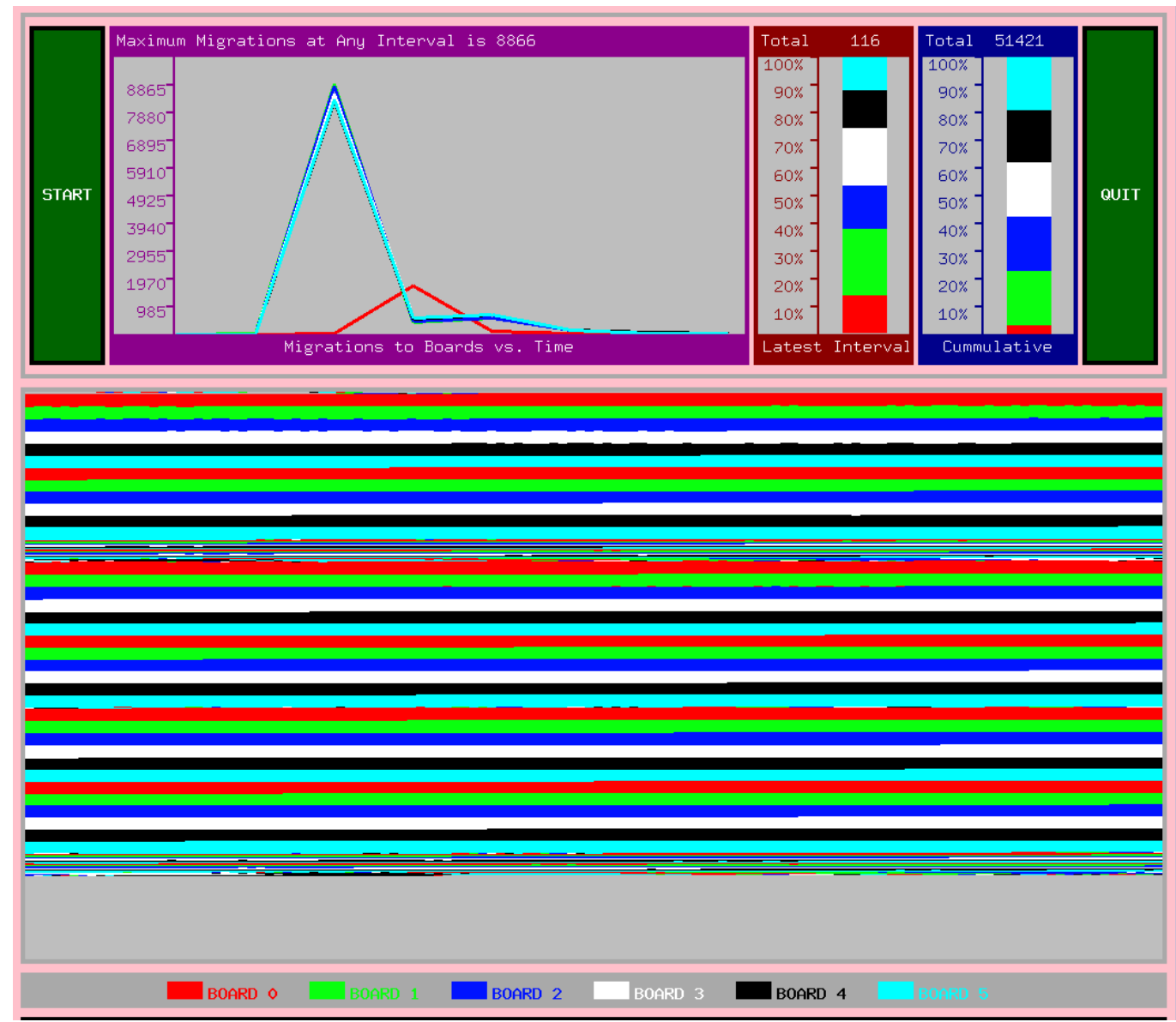

Figure 5: GUI snapshot for page placement in MG with page migration

\subsection{Simulation Experiments for other Potential Sources of Hardware Profiles}

To evaluate the effectiveness of each source of profiles for page access frequencies, we conducted a simulation study using the full system simulator Simics[12]. For our research, we chose to simulate a Sun Fire 6800 as the target cc-NUMA system. Despite its small ratio of local to remote memory latency, it allows us to compare our simulation study to the actual page migration experiments described in Section 5.2. We installed the Solaris 9 binaries on the simulated machine.

The memory subsystem of UltraSparc III processors in Sun Fire server includes five caches in their memory hierarchy, four on-chip caches and one external cache. These caches include an L1 data cache, an L1 instruction cache, a pre-fetch cache, a write cache and an L2 external cache. In addition, the memory management unit includes two data and two instruction TLBs that are accessed in parallel. In each pair of TLBs, one TLB is smaller and is used to support larger page sizes (64K-4M). 
By default, Simics does not model any cache system or memory subsystem. It uses its own internal memory representation where the memory is always up to date with the latest CPU and device transactions. However, the functionality of Simics can be extended by user-written modules[20]. To simulate the memory subsystem of UltraSparc III processors, we both modified the already available Simics modules and implemented a new timing module. We also implemented a separate module to simulate the on-chip TLBs. In addition to the memory subsystem, we also implemented a monitoring module for the data collection methods we want to evaluate. These include on-chip hardware performance monitors to gather cache miss and TLB miss information, centralized Sun Fire Link monitors to gather interconnect transactions, and our hypothetical ATC hardware to gather page access frequencies.

To investigate the effectiveness of each source of profiles in dynamic page migration, we conducted simulation experiments using the OpenMP C implementation of the NAS Parallel Benchmark suite. We chose applications with different sizes from A to B such that each application would have a similar memory footprint. Moreover, we modified the number of iterations in each application to keep the simulation time manageable. We compiled the applications using Sun's native compiler with optimizations (xopenmp=parallel) on an actual Sun Fire server and copied them to the simulated Sun Fire server.

For all experiments, as the target machine, we booted a 24-processor Sun Fire 6800 in the simulator with $12 \mathrm{~GB}$ of main memory where each locality group contains $2 \mathrm{~GB}$ main memory. The default processor type in the simulated machine is an UltraSPARC III. Since we used interactive mode to set up the simulated machine including installing the operating system, copying the compiled executables to the simulated disk, we used the default processor settings.

To quantify the benefits of using each source of profiles on dynamic page migration, we ran a series of experiments with and without page migration using the simulator. For each application, we ran the application with dynamic page migration several times varying the source of profiles. Additionally, to investigate the impact of accurate page access frequencies on the effectiveness of dynamic page migration, we also ran each application where pages are migrated based on perfect profiles. Perfect profiles are gathered by having the simulator use a full memory access history to find the references for all page references during 
the next migration interval. This allows us to quantify the cost of the less than perfect profiles produced by sampling of all page references up to the migration intervals. For the experiments with page migration, we triggered page migration at every 5 seconds like the experiments in Section 5.2.

For all experiments, we used the same simulation parameters for the simulated memory subsystem except we varied the sampling method used to gather profiles from hardware monitors. Table 5 summarizes the parameters we used in our experiments for each source of profiles to generate page access frequencies.

\begin{tabular}{|c|c|c|c|c|c|}
\hline & $\begin{array}{l}\text { Interconnect } \\
\text { Transactions }\end{array}$ & $\begin{array}{l}\text { Cache } \\
\text { Misses }\end{array}$ & $\begin{array}{c}\text { TLB } \\
\text { Misses }\end{array}$ & $\begin{array}{c}\text { TLB } \\
\text { Content }\end{array}$ & $\begin{array}{c}\text { ATC } \\
\text { Content }\end{array}$ \\
\hline Sampling Method & Centralized & \multicolumn{4}{|c|}{ Distributed } \\
\hline Sampling Interval & 512 transactions & \multicolumn{2}{|c|}{512 miss events } & \multicolumn{2}{|c|}{$16 \mathrm{~K}$ translation events } \\
\hline Local Latency & \multicolumn{5}{|c|}{$225 \mathrm{~ns}$} \\
\hline Non-Local Latency & \multicolumn{5}{|c|}{ 300ns } \\
\hline I-TLB & \multicolumn{5}{|c|}{ 128-entry, 2-way associative, 8K pages } \\
\hline D-TLB & \multicolumn{5}{|c|}{ 512-entry, 2-way associative, $8 \mathrm{~K}$ pages } \\
\hline L1 D-Cache & \multicolumn{5}{|c|}{$64 \mathrm{~KB}, 4$-way associative, 32-byte lines, 2ns hit time } \\
\hline L1 I-Cache & \multicolumn{5}{|c|}{ 32KB, 4-way associative, 32-byte lines, $2 \mathrm{~ns}$ hit time } \\
\hline L2 Cache & \multicolumn{5}{|c|}{ 8MB, 2-way associative, 512-byte lines, $16 \mathrm{~ns}$ hit time } \\
\hline
\end{tabular}

Table 5. System parameters and their values used in simulation experiments

\subsubsection{Memory Access Locality Experiments with Page Migration}

For each simulation experiment, we measured the percentage reduction in the number of non-local memory accesses in the application when memory pages are dynamically migrated compared to its original execution. We also measured the total number of pages migrated throughout the execution.

Table 6(a) presents the percentage of non-local memory accesses for the applications we tested with and without page migration. The second column presents the percentage of non-local memory accesses in the original execution. The next five columns present the percentages of non-local memory accesses when applications are run with dynamic page migration using different sources of profiles to generate page access frequencies. The last column presents the percentage of non-local memory accesses using accurate page access frequencies gathered from all actual memory accesses. For each application and source of profiles, Table 6 (a) also gives the percentage reduction in the number of non-local memory accesses with respect to the original execution of the application. 
Like Table 6 (a), Table 6 (b) presents the number of page migrations triggered when applications are run with dynamic page migration using different sources of profiles to generate page access frequencies. However, for each application, the number in parenthesis in each cell in Table 6 (b) gives the ratio of the number of page migrations triggered with respect to the number of page migrations triggered using perfect profiles. We present these ratios for a better comparison of the number of page migrations triggered for different source of profiles (Figure 6 also presents the reduction percentages in Table 6 (a))

\begin{tabular}{|c|c|c|c|c|c|c|c|}
\hline & \multirow{2}{*}{$\begin{array}{l}\text { Orig. } \\
\% \text { Non- } \\
\text { Local }\end{array}$} & \multicolumn{6}{|c|}{$\%$ of Non-Local Accesses (\% Reduction over Original) } \\
\hline & & $\begin{array}{c}\text { Intercon. } \\
\text { Trans. }\end{array}$ & $\begin{array}{l}\text { Cache } \\
\text { Misses }\end{array}$ & $\begin{array}{c}\text { TLB } \\
\text { Misses }\end{array}$ & $\begin{array}{c}\text { TLB } \\
\text { Content }\end{array}$ & $\begin{array}{c}\text { ATC } \\
\text { Content }\end{array}$ & $\begin{array}{l}\text { Perfect } \\
\text { Profiles }\end{array}$ \\
\hline BT-A & 42.0 & $29.4(\mathbf{3 0 . 1})$ & $29.1(30.9)$ & $36.9(\mathbf{1 2 . 1})$ & 30.7 (26.9) & $28.2(33.0)$ & 28.1 (33.3) \\
\hline CG-B & 79.5 & $13.7(\mathbf{8 2 . 7})$ & $11.9(\mathbf{8 5 . 0})$ & $26.6(66.5)$ & $11.8(\mathbf{8 5 . 2})$ & $11.3(\mathbf{8 5 . 8})$ & $11.7(\mathbf{8 5 . 3})$ \\
\hline FT-B & 77.0 & 66.5 (13.6) & $66.0(14.3)$ & (6.6) & $64.2(16.7)$ & $64.0(\mathbf{1 6 . 9})$ & $63.8(\mathbf{1 7 . 1})$ \\
\hline LU-B & 42.5 & 35.5 (16.5) & $34.3(\mathbf{1 9 . 3 )}$ & $46.6 \quad(-9.6)$ & $(0.8)$ & (3.3) & 33.5 (21.3) \\
\hline MG-B & 80.6 & $14.8(\mathbf{8 1 . 7})$ & $12.9(\mathbf{8 4 . 0})$ & 46.3 (42.6) & $10.3(87.2)$ & $10.2(87.3)$ & 10.0 (87.6) \\
\hline SP-B & 69.0 & $54.0(21.7)$ & 53.8 (22.0) & $62.6 \quad(9.3)$ & 55.8 (19.1) & 54.5 (21.0) & 52.9 (23.4) \\
\hline
\end{tabular}

(a) Percentage of non-local accesses for different sources of profiles

\begin{tabular}{|c|c|c|c|c|c|c|c|c|c|c|}
\hline & \multicolumn{10}{|c|}{ Number of Page Migrations Triggered (Ratio with respect to Perfect Profiles) } \\
\hline & \multicolumn{2}{|c|}{$\begin{array}{l}\text { Intercon. } \\
\text { Trans. }\end{array}$} & \multicolumn{2}{|c|}{$\begin{array}{l}\text { Cache } \\
\text { Misses }\end{array}$} & $\begin{array}{c}\text { TLB } \\
\text { Misses }\end{array}$ & \multicolumn{2}{|c|}{$\begin{array}{c}\text { TLB } \\
\text { Content }\end{array}$} & \multicolumn{2}{|c|}{$\begin{array}{c}\text { ATC } \\
\text { Content }\end{array}$} & \multirow{2}{*}{$\begin{array}{r}\begin{array}{c}\text { Perfect } \\
\text { Profiles }\end{array} \\
15,730\end{array}$} \\
\hline BT-A & 34,529 & $(2.20)$ & 31,422 & $(2.00)$ & $36,472(\mathbf{2 . 3 2})$ & 17,298 & (1.10) & 14,122 & $(0.90)$ & \\
\hline CG-B & 18,828 & $(0.97)$ & 18,920 & (0.98) & $18,524(\mathbf{0 . 9 6 )}$ & 19,823 & (1.02) & 19,308 & (1.00) & 19,344 \\
\hline FT-B & 190,313 & (1.05) & 214,605 & (1.18) & $98,320 \quad(\mathbf{0 . 5 4})$ & 156,180 & $(0.86)$ & 155,578 & $(0.86)$ & 181,632 \\
\hline LU-B & 22,88 & $(2.23)$ & 21,177 & $(2.07)$ & 19,492 (1.90) & 8,589 & $(0.84)$ & 4,897 & $(0.48)$ & 10,241 \\
\hline MG-B & 51,36 & (1.06) & 52,435 & (1.08) & $34,009 \quad(\mathbf{0 . 7 0})$ & 49,102 & (1.01) & 48,552 & $(\mathbf{1 . 0 0})$ & 48,397 \\
\hline SP-B & 35,420 & $(1.43)$ & 34,453 & (1.39) & 40,571 (1.64) & 25,035 & (1.01) & 25,233 & (1.02) & 24,814 \\
\hline
\end{tabular}

(b) Number of page migrations triggered for different sources of profiles

\section{Table 6. Results of memory locality experiments for different sources of profiles}

At first glance, it looks like dynamic page migration is effective in reducing the number of non-local memory accesses independent of the source of profiles used to gather page access frequencies. The one exception is that the number of non-local memory accesses is increased for LU only when TLB miss information is used. Overall, the reduction in the number of non-local accesses ranged from $-9.6 \%$ to $87.3 \%$. Moreover, it appears that the behavior of the different data collection techniques can broadly be grouped in to three different categories based on the number of non-local memory accesses and the number of page 
migrations triggered. In particular, the results show that using interconnect transactions performs similar to using cache misses, and using ATC content performs similar to using TLB content, and using TLB misses performs poorly compared to the others.

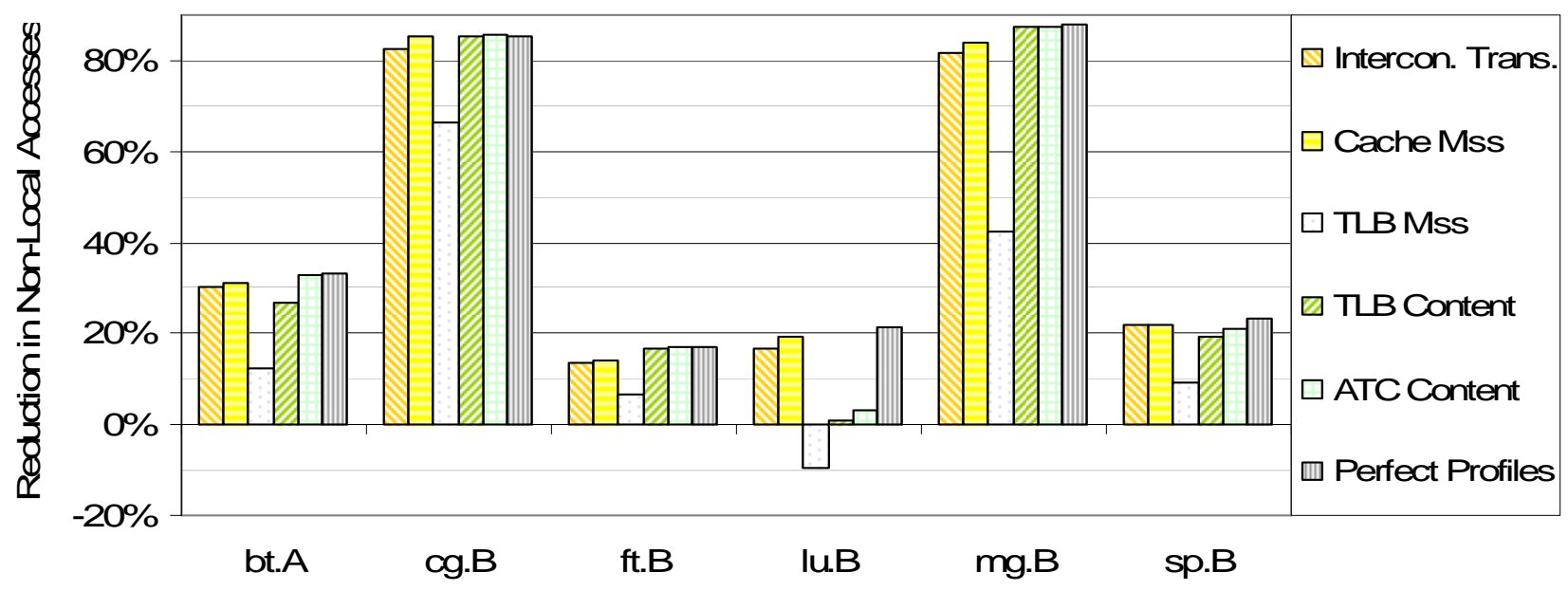

Figure 6: Percentage reduction in non-local accesses wrt. original executions

Table 6 (a) shows that when perfect profiles are used, dynamic page migration reduces the number of non-local memory accesses in the applications by $17.1-87.6 \%$. Table 6 (a) also shows that page migration using interconnect transactions reduces the number of non-local memory accesses by $13.6 \%$ to $82.7 \%$.

For some applications the reduction in the number of non-local memory accesses is slightly lower compared to the reduction percentages presented in Section 5.2.1. This is mainly due the fact that we modified the number of iterations in the applications to obtain manageable simulation times. Most of the page migrations are triggered early in the execution of these applications and during the rest of the execution they benefit from these page migrations. Thus, by reducing the number of iterations in an application, the application does not fully benefit from dynamic page migrations. However, Table 6 (a) also shows that the reduction using interconnect transactions are comparable to using perfect profiles, which indicates that using interconnect transactions is effective in approximating the actual page access frequencies.

Table 6 (a) and (b) show that using cache miss information in dynamic page migration performs slightly better compared to using interconnect transactions in terms of the reduction in the number of non-local memory accesses and the number of page migrations triggered. For the majority of applications, using cache miss information reduces the number of non-local memory accesses slightly more and triggers slightly fewer 
page migrations compared to using interconnect transactions. Moreover, the results show that using cache miss information performs closer to using perfect profiles compared to using interconnect transaction in terms of the reduction in the number of non-local memory accesses. Thus, by distributed sampling of cache misses from on-chip CPU hardware monitors in a multiprocessor, dynamic page migration can accurately generate page access frequencies in an applications and can be as effective as centralized sampling of interconnect transactions.

Table 6 (a) and (b) also show that using TLB and ATC content in dynamic page migration perform similar and they are comparable in terms of the reduction in the number of non-local memory accesses to using cache miss and interconnect transaction information for all applications except LU. In LU, they are not as effective in reducing the number of non-local memory accesses even though they trigger significantly fewer page migrations. In terms of the number of page migrations triggered, using TLB and ATC content tend to trigger fewer page migrations compared to using cache miss information and interconnect transactions. However, for $\mathrm{CG}$ and $\mathrm{MG}$ where dynamic page migration is highly effective, they trigger comparable number of page migrations.

Table 6 (a) and (b) show dynamic page migration using TLB miss information is not as effective as other sources of profiles. Even though using TLB miss information triggers fewer migrations, it is not as effective since the page access frequencies gathered from TLB miss information is not representative of page access frequencies in the applications. Moreover, dynamic page migration using TLB miss information increases the number of non-local memory accesses for LU by around $10 \%$.

Table 6 (a) also shows that using ATC content for CG performs slightly better (0.5\%) compared to using perfect profiles, which indicates that perfect profiles may not always be a perfect predictor of the future. Perfect profiles are generated using all memory accesses. If an application occasionally exhibits different memory behavior during different intervals compared to the overall behavior, perfect profiles will include profiles from these intervals which may reduce the accuracy of prediction. However, Table 6 (a) overall shows that using sources of profiles other than TLB misses performs comparable to perfect profiles, which 
also indicates that sampling of transactions does not have a significant impact on the accuracy of page access frequencies generated.

Overall, Table 6 (a) and (b) show that the sources of profiles other than using TLB miss information perform similar in terms of the reduction in the number of non-local memory accesses. More importantly, they show that cache miss profiles gathered from on-chip hardware monitors can be effectively used to guide page migrations in an application. This is particularly encouraging since such on-chip counters are included in many recent processors, and instrumentation software to access these counters are publicly available. Thus using cache miss information via distributed sampling in dynamic page migration is an easy and effective approach. Even though using TLB and ATC content performs slightly better for some applications, their use requires new hardware and system calls.

\subsubsection{Case Study: Memory Access Locality in MG}

To further investigate how dynamic page migration works using different sources of profiles for page access frequencies, we present the number of page migrations triggered versus time for MG (size B). We chose to present the results for MG since both our actual dynamic page migration approach and our simulation study have shown page migration to be most effective for this application.

Figure 7 presents the number of page migrations triggered versus time in MG for experiments with dynamic page migration using different sources of profiles. We measured the number of page migrations after each migration interval, thus the $\mathrm{x}$-axis is labeled with increasing order of migration interval.

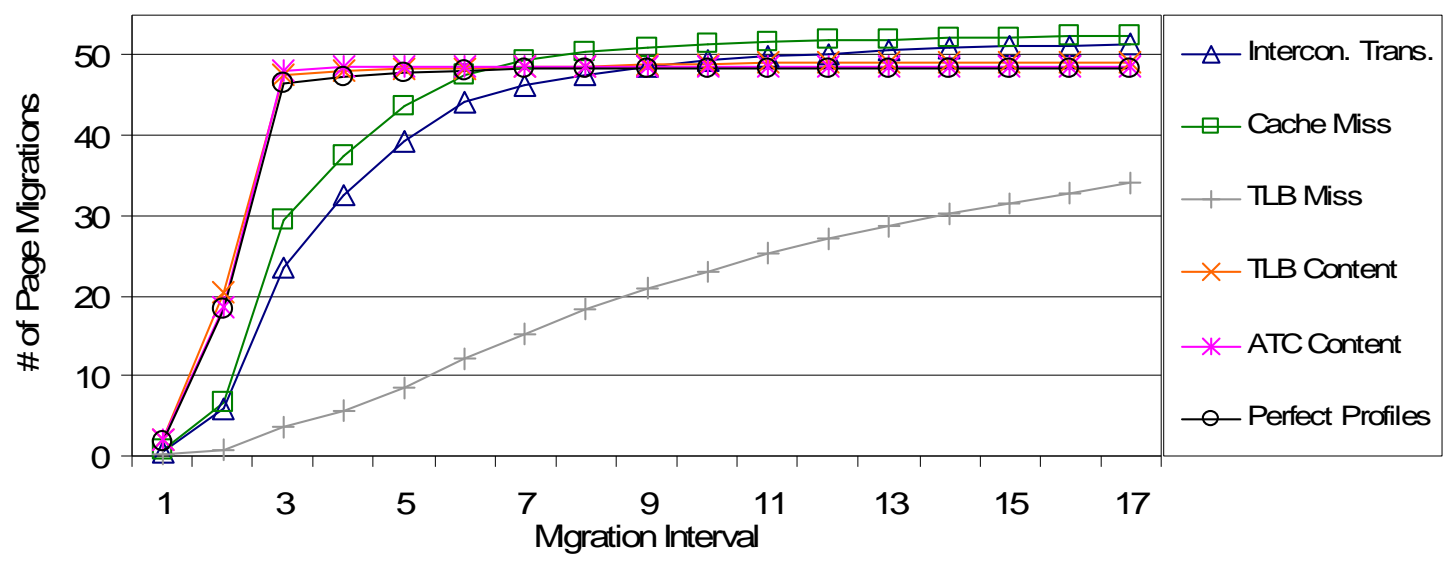

Figure 7. Number of migrations triggered by time in MG 
Figure 7 shows that when MG is run with dynamic page migration using sources of profiles other than TLB miss information, the majority of the page migrations are triggered early in the execution. Using TLB and ATC information triggers more migrations during the first seven migration intervals compared to using interconnect transactions and cache miss information but the latter sources of profiles trigger slightly more migrations in total. Overall, the number of migrations triggered in MG is comparable for all sources of profiles other than using the TLB misses. Dynamic page migration using TLB miss information triggers page migrations throughout the execution and triggers significantly fewer overall page migrations.

More importantly, Figure 7 shows that using profiles other than TLB miss information triggers a similar number of page migrations compared to using perfect profiles. However, using TLB and ATC content matches the behavior of using perfect profiles slightly better compared to using other sources of profiles.

Overall, Figure 7 shows that using TLB content and ATC content is initially more effective in reducing the number of non-local memory accesses in MG compared to using cache misses and interconnect transactions. However, by the end of execution, all sources of profiles except TLB miss information provide comparable information.

\subsubsection{Execution Times}

To investigate the impact of the reduction in the number of non-local memory accesses in the execution times of the applications, we also measured the total number of cycles spent to execute each application using different sources of profiles in the simulator. For each simulation experiment, we measured the total number of cycles spent to satisfy memory accesses.

Our simulation experiments showed that even though the total number of cycles to satisfy memory accesses is reduced by up to $16 \%$ for the applications, the impact of this reduction on the total number of cycles to execute the applications was not significant (typically about a $0.5 \%$ improvement). This is due to the fact that even though our simulator can simulate instructions executed by an application accurately, it lacks the ability to properly simulate the contention for the memory units. Moreover, in such a simulation environment it is also difficult to accurately simulate simultaneous out-of-order issue of multiple instructions by multiple processors. Thus, since our workload exhibits very low cache miss behavior, the performance 
improvement in actual memory accesses did not have a significant impact on the overall performance of the applications when the simulator executes one instruction at a time. We believe this limitation contributes to conclusions reached by previous researches that indicated limited benefit to page migration in cc-NUMA systems $[4,13,19]$.

To verify this claim, we conducted experiments where we ran MG (size B) under different page placement scenarios on an actual machine to isolate the impact of memory contention and the impact of the reduction in the number of non-local memory accesses on its execution performance.

Originally, almost all pages in MG are placed into a single memory unit which results in $80.5 \%$ of nonlocal memory accesses in its execution. Since the majority of the pages are placed on a single memory unit, the contention for this memory unit during execution is very high. To investigate the impact of memory contention on the execution performance of $\mathrm{MG}$, we ran MG under two different page placement scenarios on an actual Sun Fire 6800 server. In the first scenario, its memory pages are placed uniformly on all memory boards and in the second scenario, all of its pages are placed in a single memory unit. We measured the percentage of non-local memory accesses in MG for each run using the Sun Fire Link counters and the fraction of non-local memory accesses remained around 80\%. Our experiments show that by placing the pages in MG uniformly to all memory boards, the execution performance of MG improved by $10.2 \%$ compared to its execution where all pages are placed in a single memory unit. This indicates that simply reducing the memory contention to a single memory unit improved the execution performance of MG by $10.2 \%$. Thus, the lack of ability to accurately capture memory contention in the simulated machine partially explains why our simulation experiments did not yield improvement in the time to run the applications.

To investigate the impact of the reduction in the number of non-local memory accesses on the execution performance, we also ran MG on an actual Sun Fire server and placed all pages local to processors accessing them most at the beginning of the execution. We did this placement based on the data gathered during execution of MG using our dynamic page migration scheme. Our experiments showed that the execution performance of MG improved by $10.3 \%$ when the pages are placed in their preferred locations compared to when all pages are placed uniformly over all memory units. This improved placement resulted in an $81 \%$ 
reduction in the number of non-local memory accesses. The difficulty to accurately simulate the actual memory subsystem and latency hiding in the instruction executions using in-order cache modules in the simulator also partially explains why our simulation experiments did not yield an improvement in the total number of cycles in the applications despite the reduction in the number of non-local memory accesses.

To better evaluate the impact of the reduction in the number of non-local memory accesses on the execution times of the application, we adopted a different approach. In this approach, during simulation experiments, we recorded the actual page migrations triggered in each simulation experiment to a log file and used the log file to trigger page migrations using our dynamic page migration scheme on the actual machine. We modified our page migration system not to gather profiles from hardware monitors but instead use log files generated during simulation to guide page migrations. For each application and source of profiles, we recoded the page migrations and ran the application on the Sun Fire 6800 server where page migrations are triggered at fixed time intervals using the recoded migration entries.

Table 7 presents the percentage improvement in the execution times of the applications we tested with migration on the actual Sun Fire 6800 server using the migration logs generated during simulations. Starting with the second column, the next five columns present the percentage improvement in the applications when they are run with dynamic page migration using different sources of profiles to generate page access frequencies. The last column presents the percentage improvement using accurate page access frequencies gathered from all actual memory accesses. For each application and source of profiles in Table 7, the positive improvements in the execution performance of applications are shown in bold.

At first glance, Table 7 shows that dynamic page migration is effective at improving the execution performance of applications CG, MG and SP independent of the source of profiles used to gather page access. Dynamic page migration slightly slowed down the execution of FT for all sources of profiles and slowed down the execution of BT and LU for profiles other than TLB and ATC information. The improvement in runtime for all applications ranged from $-1.8 \%$ to $18.3 \%$. Moreover, similar to Table 6 (a) and (b), Table 7 shows that the behavior of dynamic page migration can broadly be grouped in to three different groups in terms of the improvement in the execution times of the applications. That is, Table 7 
shows that using interconnect transactions performs similar to using cache miss information, and using ATC content performs similar to using TLB content, and using TLB miss information performs differently compared to other sources of profiles. Also with our ATC hardware, we were able to improve the execution times of 5 of 6 applications versus 3 of 6 for our original centralized monitors.

\begin{tabular}{|l|r|r|r|r|r|r|}
\hline \hline & \multicolumn{6}{|c|}{ Improvement in Execution Times compared to Original } \\
\cline { 2 - 7 } & $\begin{array}{c}\text { Intercon. } \\
\text { Trans. }\end{array}$ & $\begin{array}{c}\text { Cache } \\
\text { Misses }\end{array}$ & $\begin{array}{c}\text { TLB } \\
\text { Misses }\end{array}$ & $\begin{array}{c}\text { TLB } \\
\text { Content }\end{array}$ & $\begin{array}{c}\text { ATC } \\
\text { Content }\end{array}$ & $\begin{array}{c}\text { Perfect } \\
\text { Profiles }\end{array}$ \\
\hline \hline BT-A & -1.0 & -0.8 & -1.7 & $\mathbf{0 . 2}$ & $\mathbf{0 . 8}$ & $\mathbf{0 . 9}$ \\
\hline CG-B & $\mathbf{8 . 4}$ & $\mathbf{8 . 4}$ & $\mathbf{6 . 5}$ & $\mathbf{8 . 5}$ & $\mathbf{8 . 6}$ & $\mathbf{8 . 5}$ \\
\hline FT-B & -0.2 & -1.8 & -0.1 & -2.4 & -1.3 & -1.5 \\
\hline LU-B & -0.8 & -1.0 & -0.9 & -0.1 & $\mathbf{0 . 4}$ & $\mathbf{0 . 3}$ \\
\hline MG-B & $\mathbf{1 6 . 5}$ & $\mathbf{1 5 . 8}$ & $\mathbf{1 3 . 1}$ & $\mathbf{1 8 . 1}$ & $\mathbf{1 8 . 3}$ & $\mathbf{1 8 . 0}$ \\
\hline SP-B & $\mathbf{5 . 5}$ & $\mathbf{5 . 8}$ & $\mathbf{2 . 8}$ & $\mathbf{7 . 1}$ & $\mathbf{7 . 6}$ & $\mathbf{7 . 2}$ \\
\hline \hline
\end{tabular}

Table 7. Improvement in execution performance for different sources of profiles

Table 7 shows that when perfect profiles are used, dynamic page migration improves the execution performance of the applications by $-1.5-18.0 \%$. Table 7 shows that for applications CG, MG and SP, dynamic page migration improves their execution performance independent from the source of profiles used compared to their original execution. The improvement is up to $18.3 \%$ for MG. Moreover, for these applications, using TLB content and ATC information performs slightly better compared to using interconnect transactions and cache miss information. This is due to the fact that using TLB content and ATC information tend to trigger fewer page migrations, which results in less migration overhead. More importantly, for these applications using cache miss information performs comparable if not better than using interconnect transactions. The minor differences for these applications when interconnect transactions and cache misses are used are due to differences in the reduction of the number of non-local memory accesses and the number of page migrations triggered during the execution of these applications.

Table 7 shows that using TLB content and ATC information performs slightly better for the applications BT and LU compared to using other profiles. This is due to the fact the for these applications dynamic page migration triggers fewer page migrations compared to other profiles while producing a similar reduction in the number of non-local memory accesses. Thus, migration overhead is not as much when TLB content and ATC information is used for these applications. 
Table 7 shows that for FT none of the profiles was effective in improving the execution performance. Like the experiments in Section 5.2.3, this due to the fact that the reduction in the number of non-local memory accesses for FT is only around $15 \%$ and the overhead introduced by migrations of any pages did not overcome the benefits due to improvement in memory access locality of FT.

Table 7 also shows that even though using TLB miss information triggers fewer migrations, it is not as effective in reducing the number of non-local memory accesses indicating that the page access frequencies gathered from TLB miss information is not representative of page access frequencies in the applications.

\section{Related Work}

Most processors now include hardware support for performance monitoring such as Compaq Alpha[7], Itanium from Intel[10], Sun UltraSPARC[17]. Similarly, shared-memory multiprocessors provide increasing hardware support for performance monitoring of the system interconnect such as Sun Fire Link Hardware[14]. These processors have for some time included ways to count events and trigger an interrupt when a given number of events occur. More recently, processors such as Intel Itanium 2, provide the ability to capture the addresses involved in these events such as the address of an access that misses a cache or TLB.

Noordergraaf and Zak[14] described a set of embedded hardware instrumentation mechanisms implemented for monitoring the system interconnect on Sun Fire servers. The instrumentation supports sophisticated programmable filtering of event counters. Their implementation results in a very small hardware footprint making it appropriate for inclusion in commodity hardware. Since the information gathered from these instrumentation mechanisms is based on sampling, the access frequencies of memory pages need to be approximated. Moreover, the information gathered from these instrumentation mechanisms only captures a subset of all memory accesses that also involve system interconnect for cache coherency.

Most prior page migration policies[1,11] have been in the context of non-cache-coherent NUMA multiprocessor systems. These kernel-level policies were based on page fault mechanisms and designed for multiprocessors with large remote to local latency ratios. Bolosky et al.[1] used memory reference traces to drive simulations of NUMA page placement policies. LaRowe et al.[11] modified OS memory management modules to decide whether a page will be migrated on a page fault. In contrast, this paper introduces page 
migration techniques for cache-coherent NUMA multiprocessor systems. Moreover, the page migration techniques in this paper work at user level and migrate pages using the page access frequencies gathered from embedded hardware monitors.

Chandra et al.[5] investigated the effects of different OS scheduling and page migration policies for cache-coherent NUMA systems using Stanford DASH multiprocessors. Although they mainly focused on OS scheduling policies, they also investigated page migration policies based on TLB misses. Chandra et al. reported that page migration did not improve the response time for the workloads used due to overhead incurred by the operating system.

Verghese et al.[19] studied the operating system support for page migration and replication in cachecoherent NUMA multiprocessors. They introduced a decision tree to select the action to be taken on memory pages upon cache misses. The actions taken for a page include replication, migration and freeze, depending on the threshold values used in the decision tree. Using the thresholds that gave the best results they evaluated their approach using a simulator for SGI Origin2000 multiprocessors and workload traces of cache misses in the applications. The multiprocessor system they used also had large remote to local memory latency ratios. They reported that dynamic page placements did not yield performance gains due to overhead introduced by the operating system.

Kernel-level dynamic page placement schemes are also extensively studied in the Sun WildFire systems $[4,8,13]$. The Sun WildFire system is a prototype cache-coherent NUMA architecture that is built from small number of large SMP nodes and has large remote to local latency ratios. Hagersten and Koster[8] evaluated the impact of coherent page replication and hierarchical affinity scheduling on TPC-C execution. They used excess-remote-cache-miss counts to guide page placements. Noordergraaf and Pas[13] also evaluated page migration and replication using a simple HPC application. To identify memory pages for migration, they used excess misses that indicate conflict and capacity misses in a local node's cache. They reported that using a replication-only policy yielded much better performance than policies that included migration. 
More recently, Bull and Johnson[4] studied the interactions between data distribution, migration and replication for the OpenMP applications. Although they particularly focused on a data distribution extension for OpenMP, they also studied the impact of page migration and replication. Their study also showed that page replication is more beneficial than migration. In contrast, this paper introduces a user-level page migration approach for cc-NUMA servers with small remote to local memory latency (1.34:1). Moreover, our page migration approach focuses on applications that are more likely to benefit from page migrations rather than trying to increase the overall system performance.

Most similar to our work, Marathe et al.[23] introduced a user-space hardware-assisted page placement scheme based on automated profiling. Similar to our work, the placement scheme allocates pages near processors that most frequently access that page and leverages performance monitoring capabilities of microprocessors to extract an approximate trace of memory accesses. This information is used to decide page affinity. Unlike our work, their work requires a separate profiling phase where the profiling information is gathered, which later is used to decide page affinity for the optimized run. In addition, Marathe et al. uses long-latency loads or DTLB misses as profile source using the ability of the hardware monitors to time load accesses. Similar to our results, Marathe et al. has shown that information from hardware monitors can be efficiently used to improve page placement and application performance can be improved over $20 \%$ with around $2.7 \%$ initial profiling cost.

Nikolopoulos et al.[24] introduced a user-level dynamic page migration scheme on multi-programmed NUMA shared memory multiprocessors. The algorithms exploited the idea of correlating the scheduling actions of the operating system with information obtained from dynamic monitoring of memory references. For profiling information, Nikolopoulos et al. used the per-page hardware reference counters already embedded in the underlying SGI system. These counters capture the frequency of accesses from each node to a particular page. Unlike our work, their scheme intercepts preemptions and thread migrations at user-level and uses these events as triggers for activating page migration algorithms that associate reference counting information with the nodes to or from which threads migrate. Unlike our research, their algorithms assume compiler support for identifying hot memory areas, that is, memory areas which are likely to concentrate 
excessive remote accesses and have several candidate pages for migration. They have also shown that significant performance improvements can be achieved for multi programmed workloads of OpenMP programs compared to the already existing IRIX page placement and migration mechanisms.

\section{Conclusions}

In this paper, we first introduced an automatic profile-driven page migration scheme and investigated the impact of this page migration scheme on the memory performance of multithreaded programs. We used commercially available plug in hardware monitors to profile the applications. We tested our dynamic page migration approach using the OpenMP C implementation of the NAS Parallel Benchmark suite.

Our dynamic page migration approach always reduced the total number of non-local memory accesses in the applications we analyzed compared to their original executions, by up to $90 \%$. Our page migration approach was also able to improve the execution time of the applications up to $16 \%$ compared to their original executions.

We believe the effectiveness of our page migration approach also shows the advantage of putting the page migration policy at the user level while only relying on the operating system kernel to provide the actual migration mechanism. We also believe that for page migration mechanism to be more beneficial, underlying operating system should provide means to trigger page migration without stopping the application. That is, if the user could simply request migration of a page and the underlying operating system could move the page during available idle cycles, most of the migration overhead would be hidden.

We also evaluated the effectiveness of using of several other potential sources of hardware profiles in dynamic page migration and compared their effectiveness to using profiles from centralized hardware monitors. In particular, we investigated the effectiveness of using profiles gathered from on-chip CPU monitors, the content of the processor TLBs and a hypothetical hardware feature designed specifically for dynamic page migration.

Our experiments showed that the reduction in the number of non-local memory accesses in the applications ranges up to $87.3 \%$ compared to not using page migration, which resulted in up to an $18.3 \%$ improvement in execution time. Moreover, our experiments showed that using interconnect transactions 
performs similar to using cache miss information, and using ATC content performs slightly better. However, using TLB miss information performs poorly compared to the other sources of hardware profiles.

More importantly, our experiments showed that using cache miss information performs comparable to using profiles gathered from hardware monitors specifically designed for page migration as well as perfect profiles constructed from all actual memory accesses. Our experiments demonstrated that cache miss profiles gathered from distributed on-chip hardware monitors, which are typically available in current microprocessors, can be effectively used to guide dynamic page migrations in an application. This also means software based migration could be performed on systems without the need for dedicated hardware monitors.

Our simulation experiments also demonstrated the importance of accurate simulators when benefits of dynamic page migration are evaluated in multiprocessor systems. In particular, our results indicate that lacking an accurate model for out of order execution in simulators could easily hide the benefits of page migration. We believe this limitation contributed to some of the conclusions reached by previous researches that indicated limited benefit to page migration in cc-NUMA systems[4,13,19].

\section{References}

[1] W.J. Bolosky, M.L. Scott, R.P. Fitzgerald, R.J. Fowler, A.L. Cox: NUMA Policies and Their Relation to Memory Architecture. International Conference on Architectural Support for Programming Languages and Operating Systems, Santa Clara, CA, April 1991..

[2] B.R. Buck, J.K. Hollingsworth: An API for Runtime Code Patching., International Journal of High Performance Computing Applications, v.14 n.4, p.317-329, November 2000.

[3] B.R. Buck, J.K. Hollingsworth: Using Hardware Performance Monitors to Isolate Memory Bottlenecks. ACM/IEEE conference on Supercomputing, Dallas, Texas, November 2000

[4] J.M. Bull, C. Johnson: Data Distribution, Migration and Replication on a cc-NUMA Architecture. European Workshop on OpenMP, Rome, Italy, September 2002.

[5] R. Chandra, S. Devine, B. Verghese, A. Gupta, M. Rosenblum: Scheduling and Page Migration for Multiprocessor Compute Servers. Architectural support for programming languages and operating systems, San Jose, CA, October 1994.

[6] A. Charlesworth: The Sun Fireplane System Interconnect. ACM/IEEE conference on Supercomputing, Denver, CO, November 2001.

[7] Compaq Computer Corporation: Alpha Architecture Handbook (Version 4), 1998.

[8] E. Hagersten, M. Koster: WildFire: A Scalable Path for SMPs. International Symposium on High Performance Computer Architecture, Page: 172, Orlando, FL, January 1999. 
[9] J. Heinrich: MIPS R10000 Microprocessor User's Manual (v2.0). MIPS Technologies, 1996.

[10] Intel Corporation: Intel Itanium 2 Processor Reference Manual for Software Development and Optimization, 2002.

[11] R.P. LaRowe, C.S. Ellis, L.S. Kaplan: The Robustness of NUMA Memory Management. ACM Symposium on Operating System Principles , Pacific Grove, CA, October, 1991

[12] P.S. Magnusson, M. Christensson, J. Eskilson, D. Forsgren, G. Hållberg, J. Högberg, F. Larsson, A. Moestedt, B. Werner: Simics: A Full System Simulation Platform. IEEE Computer, 35(2): 50-58, 2002.

[13] L. Noordergraaf, R. van der Pas: Performance Experiences on Sun's WildFire Prototype. ACM/IEEE conference on Supercomputing, Portland, OR, November 1999.

[14] L. Noordergraaf, R. Zak: SMP System Interconnect Instrumentation for Performance Analysis. ACM/IEEE conference on Supercomputing, Baltimore, MD, November 2002.

[15] Omni OpenMP Compiler Project: NAS Parallel Benchmarks OpenMP C Versions. http://phase.hpcc.jp/Omni/benchmarks/NPB, 2000.

[16] M.S. Squillante, E.D. Lazowska: Using Processor-cache Affinity in Shared Memory Multiprocessor scheduling. IEEE Transactions on Parallel and Distributed Systems, 4(2), 1993.

[17] Sun Microsystems: UltraSPARC III Cu User's Manual (version 1.0), 2002.

[18] M.M. Tikir, J.K. Hollingsworth: Using Hardware Counters to Automatically Improve Memory Performance. ACM/IEEE Conference on Supercomputing, Pittsburgh, PA, November 2004.

[19] B. Verghese, S. Devine, A. Gupta, M. Rosenblum: Operating System Support for Improving Data Locality on CC-NUMA Compute Servers. International Conference on Architectural Support for Programming Languages and Operating Systems, Cambridge, MA, October 1996.

[20] VirtuTech Corporation: Simics Programming Guide (v2.2.4). http://www.virtutech.com/products/, 2005.

[21] M. Woodacre, D. Robb, D. Roe, K. Feind: The SGI Altix 3000 Global Shared-memory Architecture. SGI White paper, Mountain View, CA, 2003.

[22] M. Zagha, B. Larson, S. Turner, M. Itzkowitz: Performance Analysis Using the MIPS R10000 Performance Counters. ACM/IEEE Conference on Supercomputing, Pittsburg, PA, November 1996.

[23] J. Marathe and F. Mueller. Hardware Profile Guided Automatic Page Placement for cc-NUMA Systems Symposium on Principles and Practice of Parallel Programming, New York, NY, March 2006.

[24] D. Nikolopoulos, T. Papatheodorou, C. Polychronopoulos, J. Labarta, and E. Ayguade. User-level Dynamic Page Migration for Multi-programmed Shared-memory Multiprocessors. International Conference on Parallel Programming, pages 95-103, Toronto, Canada, August 2000. 